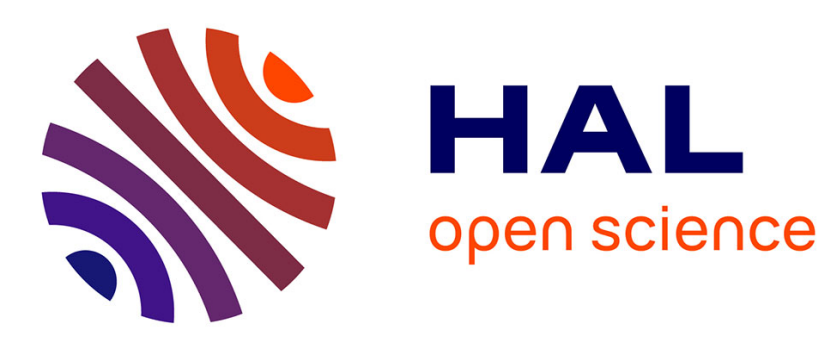

\title{
AMS-02 and its potential in the search for dark matter C. Goy
}

\section{To cite this version:}

C. Goy. AMS-02 and its potential in the search for dark matter. International Workshop on the Identification of Dark Matter 4 IDM2002, Sep 2002, York, United Kingdom. pp.507-512. in2p300012741

\section{HAL Id: in2p3-00012741 https://hal.in2p3.fr/in2p3-00012741}

Submitted on 14 Apr 2003

HAL is a multi-disciplinary open access archive for the deposit and dissemination of scientific research documents, whether they are published or not. The documents may come from teaching and research institutions in France or abroad, or from public or private research centers.
L'archive ouverte pluridisciplinaire HAL, est destinée au dépôt et à la diffusion de documents scientifiques de niveau recherche, publiés ou non, émanant des établissements d'enseignement et de recherche français ou étrangers, des laboratoires publics ou privés. 
LAPP-EXP 2002-10

December 2002

AMS-02 and its potential in the search for dark matter

\author{
C. Goy \\ LAPP-IN2P3-CNRS \\ BP. 110 - F-74941 Annecy-le-Vieux Cedex
}

$4^{\text {th }}$ International Workshop on the Identification of Dark Matter

York (UK), 2-6 September 2002 


\title{
AMS-02 AND ITS POTENTIAL IN THE SEARCH FOR DARK MATTER
}

\author{
CORINNE GOY \\ Laboratoire d'Annecy-le-Vieux de Physique des Particules. \\ F - 74941 Annecy-le-Vieux Cedex. \\ IN2P3/CNRS \\ E-mail: corinne.goy@lapp.in2p3.fr
}

This article gives a description of the AMS-02 detector, focussing on the objective of indirect searches for Dark Matter.

\section{Introduction}

The Alpha Magnetic Spectrometer, AMS-02, is a high-energy physics type of detector, scheduled for a 3 year mission on board the International Space Station. The physics goals of AMS are to search for anti-matter in cosmicrays with a sensitivity at the level of $10^{-9}$, to make high statistics measurements of the composition of charged cosmic-rays, as well as gamma-rays in the $\mathrm{GeV}$ to $\mathrm{TeV}$ energy range, and to search indirectly for the neutralino, a candidate for Dark Matter.

\section{Neutralino as DM candidates}

The presence in our universe of non-baryonic Dark Matter is now well established ${ }^{1}$, with a contribution ranging between 0.1 and 0.3 of the total energy density $\Omega$. The neutralino, arising in Super-Symmetric models as a linear combination of the super parterns of the gauge and Higgs bosons is a viable candidate for Dark Matter. In several models where R-parity is conserved, the neutralino is the lightest super-symmetric particle and is stable.

The neutralino could then be detected through the decay products of its annihilation in the galactic halo, leading to an additional spectrum of either charged particles, $p, \bar{p}, e^{+}, e^{-}, D, \bar{D}$ or $\gamma^{\prime} s$. Due to the larger background of matter in cosmic-rays, such as protons and electrons, experimentally, the search will be oriented toward anti-matter such as $\bar{p}, e^{+}, \bar{D}$ and $\gamma^{\prime} s$. 


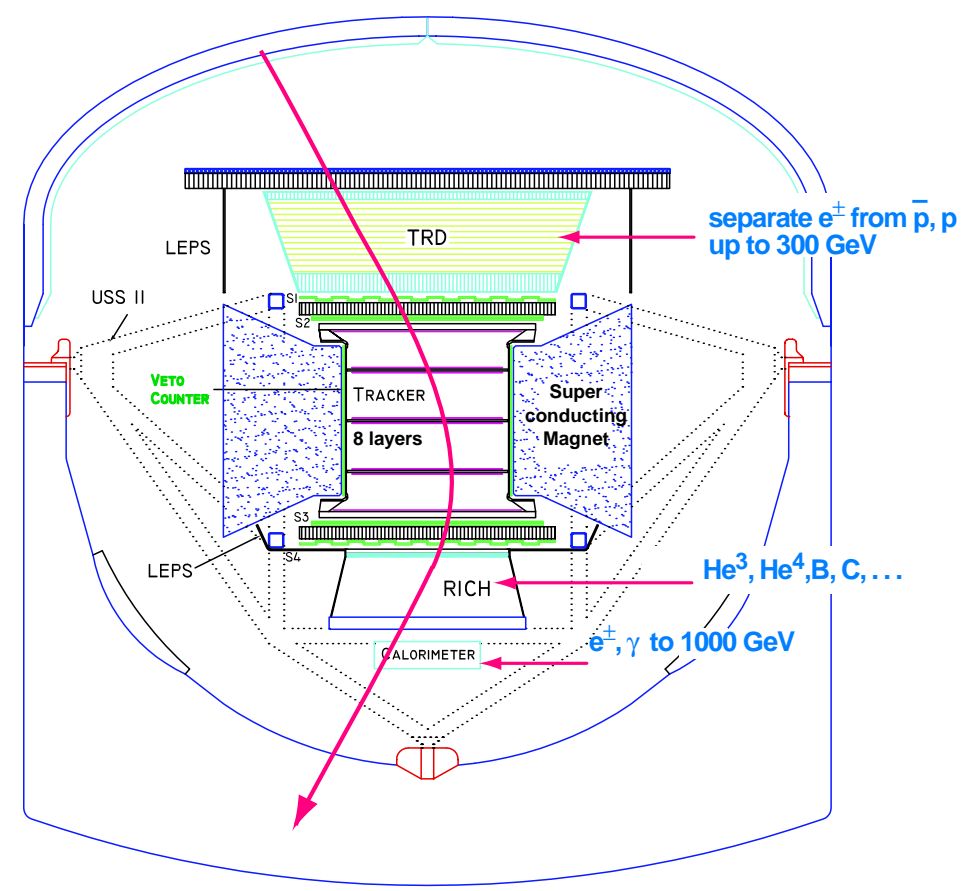

Figure 1. Schematic of AMS-02 detector.

Therefore it is essential to determine the charge of the particles and to obtain a rejection against the proton background of, e.g., at least $10^{4}$ at $200 \mathrm{GeV}$. A good energy resolution is helpful to identify a signal on top of an expected background falling as $E^{-\alpha}$ where $\alpha=2.7$ to 3 .. Identifying photons provides a complementary channel.

\section{The AMS-02 detector}

AMS-02 is designed to meet these goals as will be seen in the following subsections. A schematic of the detector is presented in figure 1. The overall detector acceptance is of the order of $0.6 \mathrm{~m}^{2} . \mathrm{sr}$, but using the calorimeter reduces it by a factor 10 . 


\subsection{The silicon tracker}

The silicon tracker is inserted in a magnetic field of $0.86 \mathrm{~T}$, created by a super-conducting magnet. It is made of 5 planes of silicon microstrip detectors, the 3 central ones being double-sided. The total surface of silicon is $6 \mathrm{~m}^{2}$. The position resolution in the bending plane is $10 \mu \mathrm{m}$.

The silicon tracker is designed to measure particle rigidity with a precision better than $15 \%$ up for hadronic particles up to TV. The resolution is $2 \%$ at $1 \mathrm{GV}$. It also provides a measurement of $\beta$ for particles with momentum less than $1.5 \mathrm{GeV}$, through $\mathrm{dE} / \mathrm{dx}$ deposition in silicon. Finally, it can reconstruct converted gammas in the range 2 to $200 \mathrm{GeV}$ with an energy resolution of $\sigma_{\mathrm{E}} / \mathrm{E}=0.025 \oplus 5.10^{-4} \times \sqrt{\mathrm{E}}(\mathrm{GeV})$ and an angular resolution of $0.018 \mathrm{deg} \oplus 0.85 \mathrm{deg} / \mathrm{E}^{2}$.

\subsection{The Time-of-flight detector}

The Time-of-flight system consists of four scintillator planes situated on either side of the tracker. With a time resolution of $150 \mathrm{ps,} \mathrm{it} \mathrm{determines}$ the direction of the crossing particle with no ambiguity at the level of $10^{-10}$. It also provides a $\mathrm{dE} / \mathrm{dx}$ measurement, and is the main component of the charged first level trigger.

\subsection{The Transition Radiation detector}

Located on top of AMS, the transition radiation detector (TRD) is designed to separate protons from positrons. It consist of 20 layers of fleece and 6 $\mathrm{mm}$ diameter straw-tubes filled with $\mathrm{Xe} / \mathrm{CO} 2$. Its main aim is to obtain a proton rejection factor of 1000 at $2 \mathrm{GeV}$ and of 100 at $200 \mathrm{GeV}$ while maintaining the positron/electron efficiency at $90 \%$. It also provides 20 additional tracking points.

\subsection{The calorimeter}

The calorimeter consists of 9 layers of lead and embedded scintillating fibres, with a thickness of $18 \mathrm{~mm}$ each. Layers are oriented alternatively along the $x$ or $y$ directions. The signal is read by 4 -anode PMTs giving a granularity of 18 samplings in depth and 0.5 Molière radius in $x$ or $y$. Due to this high granularity, a hadron rejection factor of the order of $10^{3}$ is expected from MC simulation. With a total number of 16 radiation lengths, the energy of electromagnetic particles is measured with a resolution of $\sigma(\mathrm{E}) / \mathrm{E}=0.03 \oplus 0.13 / \sqrt{\mathrm{E}}(\mathrm{GeV})$ up to approximatively $2 \mathrm{TeV}$. 
The calorimeter information is used in the positron trigger and in the stand-alone gamma trigger. Gammas in the calorimeter acceptance are reconstructed with an angular resolution of $\sigma(\Omega)=0.9 \mathrm{deg} \oplus 8.5 \mathrm{deg} / \sqrt{\mathrm{E}}$.

\subsection{The Ring Imaging Cherenkov detector}

The Ring Imaging Cherenkov detector consists of an aerogel radiator plate with a refractive index 1.05 followed by a drift space. The Cerenkow ring is detected on an array of multi-anode PMTs situated underneath. The velocity $\beta$ is measured with $0.1 \%$ precision.

Associated with the rigidity measurement in the tracker, the RICh will provide an identification of isotopes such as $B e^{9}$ and $B e^{10}$ up to an atomic number of 25 for nuclei with momentum less than $15 \mathrm{GeV} / \mathrm{N}$ and of chemical elements with $\mathrm{Z}$ less or equal 26 for momentum in $\mathrm{TeV} / \mathrm{N}$ range.

The RICh also provides an additional proton rejection factor for momentum less than $15 \mathrm{GeV}$, and rejects albedo particles at the level of $10^{-3}$.

\section{Neutralino indirect signal detection}

The search for an indirect signal of neutralino annihilations will be performed in the following channels: positrons, antiprotons, antideuterons and gammas.

\subsection{Positrons}

The AMS-02 acceptance for positrons has been estimated to be $0.15 \mathrm{~m}^{2}$.sr at $10 \mathrm{GeV}$ and $0.045 \mathrm{~m}^{2}$.sr at higher energy ${ }^{2}$. Therefore, in 3 years, the positron spectrum will be measured to $300 \mathrm{GeV}$ with a statistical precision better than $1 \%$ around $100 \mathrm{GeV}$ and $30 \%$ at $300 \mathrm{GeV}$.

\subsection{Antiprotons and antideuterons}

Recent measurements of the low energy antiprotons spectrum from BESS ${ }^{3}$ and AMS-01 ${ }^{4}$ and recent calculations of the expected secondary antiproton spectrum from conventional origins ${ }^{5}$ are now in agreement. However, the precision of the predictions is only of the order of $25 \%$ and an additional SUSY signal cannot be excluded. In figure 2, a simulated measurement of the antiproton spectrum with AMS in 3 years is presented with statistical errors ${ }^{6}$. At high energy, some SUSY models with heavy neutralino 7 predict sizeable effects in the range accessible with AMS, assuming that there is a clumpy dark halo. ${ }^{7}$. 


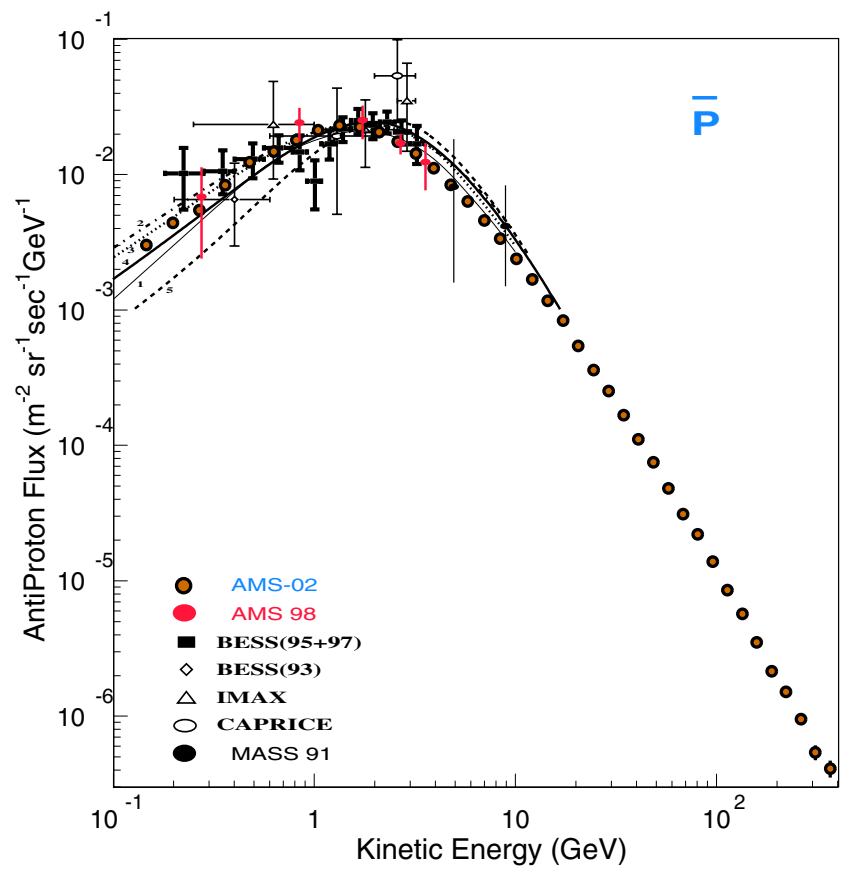

Figure 2. Simulated measurement with statistical errors of antiproton spectrum in AMS in 3 years ${ }^{6}$.Surperimposed are a compilation of antiproton measurements ${ }^{3}{ }^{4}$ and models ${ }^{3}$.

At low energy, an appealing alternative lies in the antideuteron spectrum 8. At energies below $3 \mathrm{GeV} / \mathrm{N}$, secondary antideuterons are suppressed with respect to "primary" antideuterons from supersymmetric origin. In 3 years, AMS could be sensitive to part of the SUSY parameter space.

\subsection{Gammas}

AMS is mounted on the ISS and is always pointing towards its local vertical. In a year, exposure to the galactic center is $6 \times 10^{9} \mathrm{~cm}^{2}$.s and AMS acceptance is of the order of $0.11 \mathrm{~m}^{2}$.sr when combining the tracker mode and the calorimeter mode ${ }^{2}$. AMS will be sensitive to the most cuspy models of dark halo, for both the gamma continuum and the resonance channels ${ }^{9}$. 


\section{Conclusion}

Concerning the indirect detection of dark matter, AMS will be able to perform precision measurements of the relevant cosmic-rays spectra. Combining these measurements will increase our sensitivity.

\section{References}

1. See for instance J. Peacock, these proceedings. IDM02

2. Cosmic photon and positron spectra measurements modelling with the AMS02 detector at ISS.

V. Choutko, G. Lamanna and A. Malinin.

Int. J. Mod. Phys. A17, 1817 (2002)

3. T. Maeno et al. Ap. Phys 16121 (2001). and references therein.

4. AMS Collaboration.

Phys. Rep. 366(2002) to come.

5. Antiprotons from spallation of cosmic rays on interstellar matter.

F. Donato et al. astro-ph/0103150

6. AntiProtons Yields on AMS02, a preliminary study. V. Choutko. Space Part 2002 May 16, 2001, Elba

7. Signatures of exotic physics in antiproton cosmic ray measurements. P. Ullio. astro-ph/9904086

8. Antideuterons as a Signature of Supersymmetric Dark Matter. F. Donato et al. Phys. Rev. D 62043003 (2000).

9. Pulsars, Blazars and Dark Matter with AMS. M. Pohl Int. J. Mod. Phys.A17, 1809 (2002) 